\title{
Diagnóstico situacional da consulta farmacêutica na rede básica de saúde do Município de Belém, Estado do Pará, Brasil
}

Situacional diagnosis of consultation in the basic health network in the Municipality of Belém, State of Pará, Brazil

Diagnóstico situacional de la consulta farmacêutica em la red básica de salud del Município de Belém, Estado de Pará, Brasil

\author{
Maria Lucia Dias Tavares \\ ORCID: https://orcid.org/0000-0002-8039-7129 \\ Universidade Federal do Pará, Brasil \\ E-mail: tavaressol123@gmail.com \\ Valdenira Gonçalves da Silva \\ ORCID: https://orcid.org/0000-0002-9983-4122 \\ Universidade Federal do Pará, Brasil \\ E-mail:vgs_farmac@yahoo.com.br \\ Hayllen Mayara Santos Gonçalves Rocha \\ ORCID: https://orcid.org/0000-0001-9429-9020 \\ Universidade Federal do Pará, Brasil \\ E-mail: hayllenmayara@yahoo.com.br \\ Ana Paula Bastos Ferreira Vieira \\ ORCID: https://orcid.org/0000-0001-8936-7420 \\ Universidade Federal do Pará, Brasil \\ E-mail: anabastos02@gmail.com \\ Priscila de Nazaré Quaresma Pinheiro \\ ORCID: https://orcid.org/0000-0001-5502-1018 \\ Universidade Federal do Pará, Brasil \\ E-mail: priscilapcr4@gmail.com \\ Marcos Valério Santos da Silva \\ ORCID: https://orcid.org/0000-0002-7824-0042 \\ Universidade de São Paulo, Brasil. \\ E-mail: marcossilva@ufpa.br \\ Marcieni Ataide de Andrade \\ ORCID: https://orcid.org/0000-0001-5875-695X \\ Universidade de São Paulo, Brasil \\ E-mail: marcieniandrade@gmail.com
}

\begin{abstract}
Resumo
Objetivou-se realizar o diagnóstico situacional da consulta farmacêutica nas Unidades Básicas de Saúde (UBS) do municipio de Belém. Trata-se de um estudo transversal de caráter descritivo com abordagem qualitativa, realizado através de coleta de dados, no período de julho a agosto de 2020, por meio de aplicação de questionário adaptado, embasado no instrumento utilizado na Pesquisa Nacional sobre o Acesso, Utilização e Promoção do Uso Racional de Medicamentos (PNAUM), o qual foi composto por dezoito (18) perguntas abertas e fechadas, acerca das atividades de ações gerenciais e ações assistenciais, geridas pelos farmacêuticos nas UBS, buscando obter informações sobre o cenário real das atividades exercidas por esses profissionais, bem como, identificar a sua atuação clínica de modo mais abrangente e de que forma é realizada na rotina de trabalho. Dentre os resultados, verificou-se que somente três UBS desenvolvem práticas relacionadas aos serviços clínicos farmacêuticos. Entre os motivos para a ausência dessa prática nas demais UBS, identificou-se a falta de infraestrutura, a deficiência de recursos humanos e indisponibilidade de tempo do profissional farmacêutico. A proposta executada é uma estratégia inicial para posterior implantação do serviço da consulta farmacêutica na rede municipal de saúde de Belém, haja vista as evidências por meio do diagnóstico situacional que comprovam a ausência do serviço de modo padronizado.

Palavras-chave: Farmácia clínica; Serviços clínicos farmacêuticos; Cuidado farmacêutico; Consulta farmacêutica; Atenção primária à saúde.
\end{abstract}

\footnotetext{
Abstract

The objective was to carry out the situational diagnosis of the pharmaceutical consultation in the Basic Health Units (BHU) in the city of Belém. This is a cross-sectional study of a descriptive character with a qualitative approach, carried
} 
out through data collection, from July to August 2020, through the application of an adapted questionnaire, based on the instrument used in the National Survey on Access, Use and Promotion of Rational Use of Medicines (PNAUM), which was composed of eighteen (18) open and closed questions about the activities of management actions and assistance actions, managed by pharmacists in the UBS, seeking to obtain information about the real scenario of the activities performed by these professionals, as well as, to identify their clinical performance in a more comprehensive way and how it is performed in the work routine. Among the results, it was found that only three UBS develop practices related to clinical pharmaceutical services. Among the reasons for the absence of this practice in the other UBS, the lack of infrastructure, the lack of human resources and the unavailability of time by the pharmaceutical professional were identified. The executed proposal is an initial strategy for the subsequent implementation of the pharmaceutical consultation service in the municipal health network in Belém, considering the evidence through the situational diagnosis that proves the absence of the service in a standardized way.

Keywords: Clinical pharmacy; Pharmaceutical clinical services; Pharmaceutical care; Pharmaceutical consultation; Primary health care.

\section{Resumen}

El objetivo fue realizar el diagnóstico situacional de la consulta farmacéutica en las Unidades Básicas de Salud (UBS) de la ciudad de Belém. Se trata de un estudio transversal de carácter descriptivo con abordaje cualitativo, realizado mediante recolección de datos, de julio a agosto. 2020, mediante la aplicación de un cuestionario adaptado, basado en el instrumento utilizado en la Encuesta Nacional de Acceso, Uso y Promoción del Uso Racional de Medicamentos (PNAUM), el cual estuvo compuesto por dieciocho (18) preguntas abiertas y cerradas sobre la actividades de acciones de gestión y acciones asistenciales, gestionadas por farmacéuticos en la UBS, buscando obtener información sobre el escenario real de las actividades realizadas por estos profesionales, así como, identificar de forma más integral su desempeño clínico y cómo se realiza en la rutina laboral. . Entre los resultados, se encontró que solo tres UBS desarrollan prácticas relacionadas con los servicios farmacéuticos clínicos. Entre las razones de la ausencia de esta práctica en las otras UBS, se identificaron la falta de infraestructura, la falta de recursos humanos y la falta de disponibilidad de tiempo por parte del profesional farmacéutico. La propuesta ejecutada es una estrategia inicial para la posterior implementación del servicio de consulta farmacéutica en la red municipal de salud de Belém, considerando la evidencia a través del diagnóstico situacional que acredite la ausencia del servicio de manera estandarizada.

Palabras clave: Farmacia clínica; Servicios farmacéuticos clínicos; Atención farmacêutica; Consulta farmacêutica; Atención primaria de salud.

\section{Introdução}

No Brasil, pode-se dizer que ao longo do tempo, ocorreram uma série de marcos legais com objetivos de reestruturar a Assistência Farmacêutica (AF), desde a sua incorporação no âmbito do Sistema Único de Saúde (SUS) como política pública, voltados para o acesso da população a medicamentos com qualidade, eficácia e segurança comprovadas, além da promoção do uso racional de medicamentos (Costa et al., 2017). Como exemplos, pode-se citar a Política Nacional de Medicamentos (PNM), em 1998, a Política Nacional da Assistência Farmacêutica (PNAF) em 2004, entre outras (Brasil; Freitas et al., 2016). Mattos (2015) em seu estudo esclarece que essas políticas em sua totalidade visam à integração, reorientação, organização e qualificação, objetivando promover melhorias das condições da $\mathrm{AF}$ em todos os níveis de atenção à saúde.

Em 2017, foi aprovada uma nova revisão nas políticas públicas nacionais para o fortalecimento da AF, destacando-se a Política Nacional de Atenção Básica (PNAB) pela portaria $n^{\circ} 2.436$, estabelecendo novas diretrizes para a organização dos serviços da AF no SUS, ampliando e qualificando o acesso da população aos medicamentos, bem como, fortalecendo o cuidado em saúde ofertado aos usuários na Atenção Primária à Saúde (APS) ou Atenção Básica à Saúde (ABS), termo também muito utilizado por alguns autores brasileiros na literatura (Araújo, S. et al., 2017; Brasil, 2017, 2019; Barros et al., 2020).

A APS é considerada a principal porta de entrada do usuário ao sistema de saúde, tendo um papel essencial na melhoria do cuidado à saúde dos cidadãos, devido ao seu potencial resolutivo e baixo custo. Desse modo, a AF exerce um papel importante na APS, complementando e aprimorando as ações e serviços de saúde, por meio da atividade farmacêutica, buscando assegurar o acesso e promoção do uso racional de medicamentos (Arruda et al., 2015; Araújo, S. et al., 2017; Luquetti et al., 2017; Brasil, 2019). Entretanto, para que o profissional farmacêutico possa realizar suas atividades na APS, deve cumprir com as diretrizes 
propostas pela PNAF que são aplicadas por meio dos Serviços farmacêuticos (Sefar) (Brasil, 2014; Cff, 2016a; Araújo, S. et al., 2017).

Os Sefar envolvem um conjunto de ações coordenadas pelo farmacêutico que visam contribuir para prevenção de doenças, promoção à saúde, tendo o medicamento como um dos elementos essenciais, contribuindo para seu acesso e uso racional (Pereira et al., 2015; Cff, 2016a; Araújo, S. et al.; Araújo, P. et al., 2017). Estes serviços na APS, se dividem em ações gerenciais e ações clínico-assistenciais (Araújo, S. et al., 2017; Brasil, 2019).

As ações gerenciais se constituem em atividades voltadas principalmente para a logística do medicamento. Essas atividades envolvem as etapas de seleção, programação, armazenamento, distribuição, gestão de pessoas, além de darem suporte a prescrição e dispensação (Pereira et al., 2015).

As ações clínico-assistenciais, também denominadas por outros autores, na literatura, como cuidado farmacêutico, serviços clínicos farmacêuticos, atenção farmacêutica e consulta farmacêutica, se caracterizam pela ação integrada do farmacêutico com a equipe de saúde, dos quais estão relacionadas ao cuidado do paciente (Araújo, S. et al.; Araújo, P. et al., 2017; Barros, 2020). Essas ações envolvem as atividades de dispensação, orientação farmacêutica, acompanhamento farmacoterapêutico, reconciliação de medicamentos, entre outros, com a finalidade de efetivar o tratamento individual ou coletivo, por meio da gestão clínica do fármaco, a fim de garantir o uso correto de medicamentos e consequentemente promover a saúde da população (Cff, 2016a; Araújo, S. et al.; Araújo, P. et al., 2017; Silva et al., 2018; Barros, 2020). Neste contexto, o farmacêutico contemporâneo é responsável por promover diversas ações de cuidado ao paciente, propondo intervenções conforme a sua necessidade de saúde e promovendo melhores resultados na farmacoterapia (Cff, 2016b; Silva et al., 2018; Brasil, 2019; Barros, 2020).

A prestação dos Sefar desenvolvidos pelo farmacêutico, na APS, é amparada e consolidada por diversos marcos regulatórios, para que este, possa exercer com afinco e segurança suas atribuições. Dentre estas regulações, destacam-se as resoluções: a RDC n 499/2008 do Conselho Federal de Farmácia (CFF), a qual dispõe sobre a prestação de serviços farmacêuticos, em farmácias e drogarias e ressalta que somente o farmacêutico poderá prestar estes serviços e, a RDC n 44/2009 da Agência Nacional de Vigilância Sanitária (ANVISA), que aprovam as Boas Práticas Farmacêuticas, definindo dimensões, mobiliário e infraestrutura compatíveis com a prestação de serviços farmacêuticos a serem oferecidos aos pacientes (Cff, 2016b; Dal Molin et al., 2018). Além disso, o CFF, posteriormente, publicou duas resoluções, em 2013, a RDC n585, que regulamenta as atribuições clínicas do farmacêutico, o qual é apto a promover saúde, prestar serviços assistenciais, com foco na efetividade do tratamento e segurança do paciente e, a RDC ${ }^{\circ} 586$ que regulamenta a prescrição farmacêutica de medicamentos e outros produtos com finalidade terapêutica, cuja dispensação não exija prescrição médica (Cff, 2016a; Freitas et al., 2016; Nicoletti \& Ito, 2018). Ao encontro dessas resoluções, foi publicada em 2014, a Lei Federal n ${ }^{\circ} 13.021$, reforçando a prática dos serviços farmacêuticos, reconhecendo as farmácias e drogarias como um estabelecimento de saúde, ao instituir obrigações legais voltadas à prestação de cuidado direto ao paciente. Sendo assim, essa lei apresenta um grande avanço no desenvolvimento dos serviços farmacêuticos nas farmácias brasileiras, enfatizando o cuidado farmacêutico centrado ao usuário, garantindo dessa forma, uma assistência farmacêutica integral e segura, de forma a promover o uso racional de medicamentos e contribuir para a otimização da farmacoterapia (Cff, 2016b; Dal Molin et al., 2018).

Portanto, segundo as legislações citadas, os farmacêuticos ao desenvolverem à prática dos Sefar, promovem à saúde, previnem e monitoram os eventos adversos, intervêm e contribuem na prescrição de medicamentos, atuando individualmente ou em conjunto com outros profissionais de saúde, com a finalidade de obter resultados clínicos positivos, promovendo dessa forma, uma melhor qualidade de vida aos pacientes (Pereira et al., 2017).

Podemos conceituar a Farmácia Clínica (FC) como área da farmácia voltada para à prática do uso racional do medicamento, na qual o farmacêutico atua em conjunto com uma equipe multiprofissional, prestando cuidado centrado ao 
paciente, visando a prevenção e resolução de problemas relacionados à farmacoterapia, de forma efetiva e humanizada (Lima et al., 2018; Rossignoli et al., 2019).

Vale ressaltar que a Consulta Farmacêutica (CF) é de suma importância para a prática da farmácia clínica, sendo esta, definida pelo contato entre o farmacêutico e o paciente, objetivando os melhores resultados na farmacoterapia, na qual pode ser provida de diferentes serviços e procedimentos, conforme a necessidade dos usuários (Cff, 2016a; Lansing et al., 2017; Brasil, 2019). Essa prática é desenvolvida em etapas, para tal, o profissional farmacêutico utiliza como método clínico, a técnica da entrevista, com a finalidade de coletar informações sobre o paciente e elaborar um plano de cuidado. Um dos métodos mais utilizados na prática clínica para coleta de dados é o $\operatorname{SOAP}(\mathrm{S}=$ subjetivo, $\mathrm{O}=$ objetivo, $\mathrm{A}=$ avaliação e $\mathrm{P}=$ plano). Segundo Lima e colaboradores (2018), "o SOAP é uma metodologia de raciocínio clínico para evolução em prontuário que padroniza o registro tornando-o claro, completo e conciso". Sendo assim, esse método visa fornecer ao farmacêutico clínico subsídios para a realização de um atendimento mais eficiente e seguro, voltado ao cuidado integrado e contínuo ao paciente (Araújo, S. et al., 2017; Cruz et al., 2020).

Diante do exposto, pode-se ressaltar que a implantação dos Sefar ainda é um desafio no município de Belém, fato este, constatado nos estudos de Soler e colaboradores (2017), que demonstraram a falta da gestão do cuidado farmacêutico na Rede Municipal de Saúde. De acordo com Barbosa (2018), o CFF em parceria com a Secretaria Municipal de Saúde e Meio Ambiente (SESMA) do município de Belém, realizou a capacitação dos profissionais farmacêuticos, através do curso de Cuidado Farmacêutico no SUS, o qual visava ampliar os atendimentos clínicos e implantar consultórios farmacêuticos nas Unidades Básicas de Saúde (UBS).

No entanto, ainda não se evidencia na literatura, a atuação clínica dos farmacêuticos nas UBS de Belém, de forma oficializada, o que ratifica a imprescindibilidade da realização do diagnóstico situacional da consulta farmacêutica na rede básica de saúde do município de Belém-Pa.

\section{Metodologia}

Realizou-se um estudo transversal de caráter descritivo, apresentando uma abordagem de natureza qualitativa. Essa abordagem, segundo Pereira, et al. (2018), trata-se de um método “[...] a interpretação por parte do pesquisador com suas opiniões sobre o fenômeno em estudo. Neles a coleta de dados muitas vezes ocorre por meio de entrevistas com questões abertas". Como instrumento para coleta de dados utilizou-se o questionário, a fim de se obter informações específicas, referentes aos mais diversos aspectos envolvidos na pesquisa, para uma melhor apreciação do conteúdo apresentado no trabalho.

A coleta de dados ocorreu no período de julho a agosto de 2020, realizado no município de Belém-Pá, tendo como foco as UBS vinculadas à rede SESMA, distribuídas em seus respectivos Distritos de Saúde, conforme cadastradas no portal da Prefeitura Municipal de Belém [PMB] (2020). As agências distritais segundo o portal da Secretaria Municipal são divididas em seis (06) coordenações de Saúde: a Coordenação de Saúde de Mosqueiro (DAMOS) composta por três (03) UBS; Coordenação de Saúde da Sacramenta (DASAC) composta por cinco (05) UBS; Coordenação de Saúde do Benguí (DABEN) composta por sete (07) UBS; Coordenação de Saúde do Guamá (DAGUA) composta por seis (06) UBS; Coordenação de Saúde do Entroncamento (DAENT) composta por seis (06) UBS; Coordenação de Saúde de Icoaraci /Outeiro (DAICO/ DAOUT) composta por cinco (05) UBS, totalizando trinta e duas (32) UBS cadastradas.

Os critérios de inclusão à aplicação do questionário aos farmacêuticos nas UBS foram exclusivamente de tipologia Atenção Básica (AB) e as UBS que prestam atendimento de Urgência e Emergência e Ambulatoriais de tipologia (MISTA), visto que, essas Unidades ao desenvolverem suas atividades, prestam serviços ambulatoriais de baixa complexidade, sendo o foco do presente estudo. Já os critérios de exclusão foram: as UBS de tipologia exclusivamente Urgência e Emergência (U/E), 
haja vista, que essas Unidades ao desenvolverem suas atividades à comunidade, prestam serviços hospitalares de média e alta complexidade, e a Estratégia de Saúde da Família (ESF), por não possuir o profissional farmacêutico em sua equipe multiprofissional, no momento da realização da pesquisa.

A elaboração do questionário foi embasada no instrumento utilizado na Pesquisa Nacional sobre o Acesso, Utilização e Promoção do Uso Racional de Medicamentos (PNAUM), elaborado pelo Ministério da Saúde (MS), e foi adaptado para o objetivo do presente estudo (Brasil, 2016b). O instrumento elaborado foi composto por dezoito (18) perguntas abertas e fechadas, acerca das atividades de ações gerenciais e ações assistenciais, geridas pelos farmacêuticos nas UBS, buscando obter informações sobre o cenário real das atividades exercidas por esses profissionais, bem como, identificar a sua atuação clínica de modo mais abrangente e de que forma é realizada na rotina de trabalho. O mesmo foi dividido em três sessões, para uma maior abrangência do tema: I-Perfil sociodemográfico; II-Dispensação de medicamentos e sobre atividades de natureza clínico-assistenciais e III Legislação.

A aplicação do questionário aos farmacêuticos vinculados às UBS foi realizada por meio de visita programada, conforme a disponibilidade dos profissionais, participando da pesquisa apenas um profissional de cada UBS. Dentre as trinta e duas UBS cadastradas e visitadas, apenas vinte e sete estavam aptas a participarem da pesquisa, conforme a aplicação dos critérios de inclusão e exclusão. No entanto, houve recusa de duas UBS, resultando na aplicação de vinte e cinco questionários $(\mathrm{N}=25)$.

Os dados coletados e analisados, foram tabulados e representados por meio de gráficos, quadros e tabelas, empregandose o programa Microsoft Excel ${ }^{\circledR} 2013$.

Este estudo foi submetido à Plataforma Brasil - Comitê de Ética da Universidade Federal do Pará - UFPA para apreciação, obtendo-se parecer favorável conforme CAAE $n^{\circ}$ 23640019.0.0000.0018. Foi apresentado a cada entrevistado o Termo de Consentimento Livre e Esclarecido (TCLE).

\section{Resultados e Discussão}

No Município de Belém, cada UBS pertence a um grupo de Coordenação de Saúde, sendo devidamente cadastrada de acordo com sua área distrital. Do total das trinta e duas (32) UBS cadastradas verificou-se que $68,75 \%$ prestam serviços da tipologia $\mathrm{AB}, 15,63 \%$ prestam serviços de atendimento da tipologia MISTA, 6,25 \% somente serviços de U/E e 9,38\% são ESF, conforme descrito na Tabela 1 . 
Tabela 1 - Distribuição quantitativa de UBS em Distritos de Saúde por Tipologia de atendimento, Belém-Pará, 2020.

\begin{tabular}{|c|c|c|c|c|c|c|}
\hline \multirow[b]{2}{*}{ Distrito } & \multicolumn{4}{|c|}{ Tipologia } & \multirow[b]{2}{*}{$\begin{array}{c}\text { TOTAL DE UBS / } \\
\text { DISTRITO }\end{array}$} & \multirow[b]{2}{*}{$\%$} \\
\hline & $\mathbf{A B}$ & MISTA & $\mathbf{U} / \mathbf{E}$ & ESF & & \\
\hline DAMOS & 01 & 01 & 01 & 0 & $\mathrm{O} 3$ & 9,38 \\
\hline DASAC & 05 & 0 & 0 & 0 & O5 & 15,63 \\
\hline DABEN & 05 & 01 & 01 & 0 & 07 & 21,88 \\
\hline DÁGUA & 04 & 01 & 0 & 01 & 06 & 18,75 \\
\hline DAENT & 04 & 01 & 0 & 01 & 06 & 18,75 \\
\hline DAICO/ DAOUT & 03 & 01 & 0 & 01 & 05 & 15,63 \\
\hline $\begin{array}{l}\text { Total das UBS } \\
\text { (SESMA) }\end{array}$ & 22 & 05 & 02 & 03 & 32 & 100 \\
\hline
\end{tabular}

Fonte: Autores.

Ao se analisar os tipos de serviços prestados por essas UBS, somente 84,37\% (27) foram consideradas aptas à aplicação do questionário, por prestarem serviços ambulatoriais de baixa complexidade: as Unidades de tipologia AB e MISTA. Já, 15,63\% (05) das UBS foram consideradas inaptas: as Unidades de tipologia U/E e ESF, conforme a aplicação dos critérios de inclusão e exclusão. Contudo, houve recusa de participação na pesquisa de duas (02) UBS, resultando na aplicação de vinte e cinco questionários $(\mathrm{N}=25)$.

Em relação às atividades executadas pelos profissionais farmacêuticos nas UBS, observou-se que ações gerenciais ainda são desenvolvidas em maior percentual em relação às atividades de ações clínico-assistenciais (Gráfico 1).

Gráfico 1 - Atividades realizadas pelos profissionais farmacêuticos nas UBS (N=25) no Município de Belém, 2020.

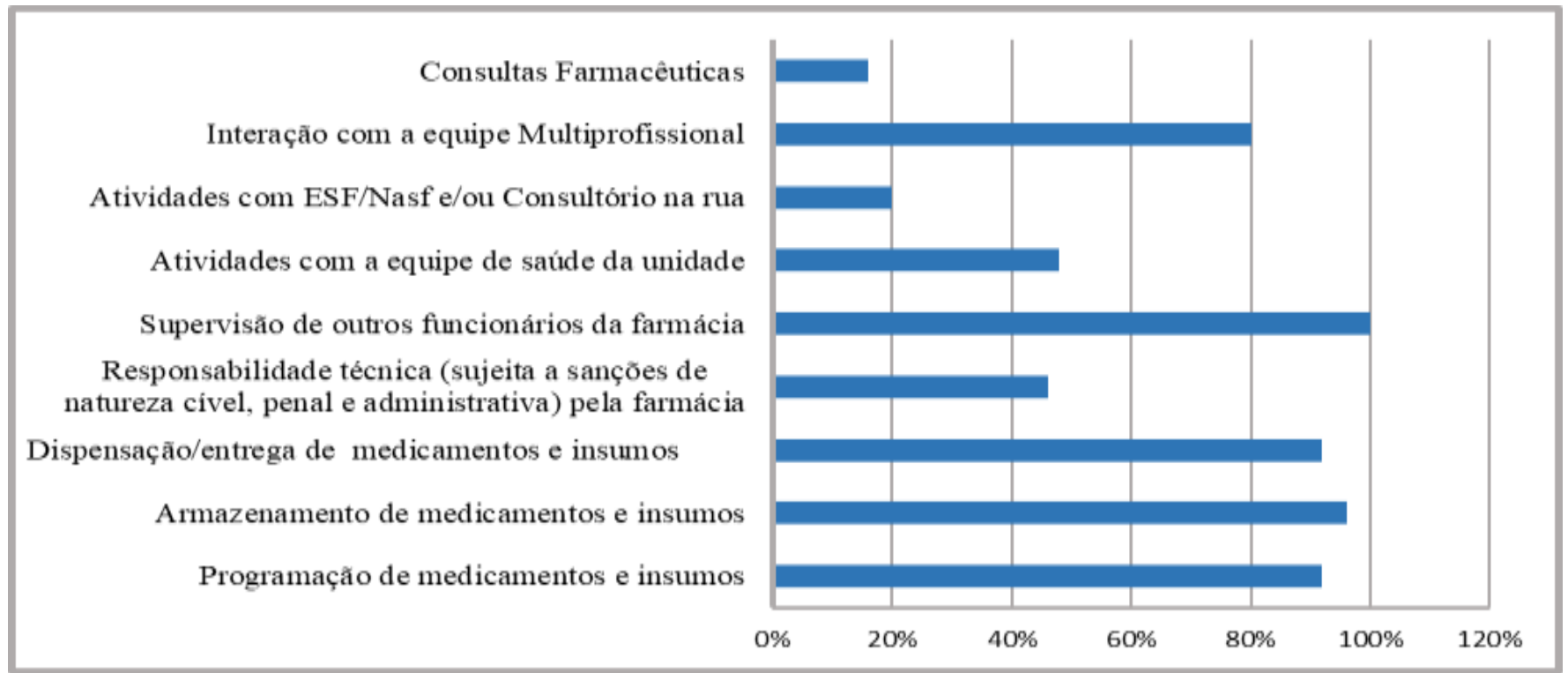

Fonte: Autores. 
De acordo com o Gráfico 1, observa-se que todos os profissionais entrevistados (100\%) realizam atividades de supervisão de outros funcionários da farmácia, assim como é disponibilizado $96 \%$ da sua carga horária ao armazenamento, e 92\% dedicam-se à gestão de programação, dispensação/entrega de medicamentos e insumos. Já a atividade de atuação clínica, a consulta farmacêutica é desenvolvida em menor percentual, apenas $16 \%$.

Observou-se em um estudo (Projeto-Piloto) sobre cuidados farmacêuticos na atenção básica, realizado em fevereiro de 2014, no município de Curitiba-PR, que o tempo médio destinado às atividades de gerenciamento de medicamentos, representou aproximadamente $80 \%$ da carga horária mensal dos farmacêuticos do NASF, enquanto às atividades clínico-assistenciais, incluindo a dispensação e consulta farmacêutica representavam próximo de $20 \%$ de sua carga horária (Brasil, 2015). No estudo de Araújo e colaboradores (2017), consta que as atividades de ação clínico-assistenciais desempenhadas pelo profissional farmacêutico na atenção básica, ainda são incipientes no Brasil e ocorrem em proporções desiguais em todas as regiões. Nesse contexto, observa-se que a prática clínica, ainda é um desafio em toda rede municipal de saúde do município de Belém, isto é, as atividades executadas pelo profissional farmacêutico, consistem em maior proporção nas ações administrativas com pouca participação no cuidado efetivo aos usuários. Isso pode ser atribuído à falta de um espaço adequado, recursos humanos insuficientes e pela predominância no processo de trabalho deste profissional, ainda estar ligado ao paradigma técnico, centrado na logística de medicamentos (Akerman \& Freitas, 2017).

Para a realização da gestão da AF nas UBS, estes profissionais utilizam o Sistema Nacional denominado Hórus, onde são lançadas todas as atividades produzidas pelo farmacêutico, tais como: controle de estoque, dispensação de medicamentos, acompanhamento do histórico da dispensação, armazenamento de medicamentos e insumos estratégicos, entre outras atividades (Costa \& Nascimento, 2012). Tal sistema é alimentado com dados de forma contínua e monitorado pela Referência Técnica de Medicamentos vinculada a Divisão de Recursos de Materiais (DRM) na SESMA, onde é realizado a análise e gerenciamento do ciclo logístico de medicamentos na APS. Todavia, podem ocorrer falhas e/ou o não preenchimento correto no sistema de informações, suscitando na deficiência da programação, seleção, controle de estoque e distribuição, do qual trará impacto direto na dispensação dos medicamentos aos usuários (Andreoli \& Dias, 2015).

Em relação à falta de medicamentos nos últimos três meses nas UBS, bem como, a (s) conduta (s) adotada (s) pelo profissional farmacêutico no contexto da falta dos medicamentos, está descrita na Tabela 2. 
Tabela 2 - Falta de medicamentos nos últimos três meses x Conduta (s) adotado (s) pelo farmacêutico frente ao usuário nas UBS (N=25), Belém-Pará, 2020.

\begin{tabular}{|c|c|c|}
\hline Falta de medicamentos (há três meses) & $\mathbf{N}$ & $\%$ \\
\hline Sempre & 10 & 40 \\
\hline Repetidamente & 09 & 36 \\
\hline Às vezes & 06 & 24 \\
\hline \multicolumn{3}{|l|}{ Raramente } \\
\hline \multicolumn{3}{|l|}{ Nunca } \\
\hline \multicolumn{3}{|l|}{ Conduta adotado pelo profissional ao usuário } \\
\hline Informa que não tem o medicamento & 20 & 80 \\
\hline Buscam informação sobre a disponibilidade em outra UBS & 15 & 60 \\
\hline Orienta ao usuário a procurar Aqui Tem Farmácia Popular & 19 & 76 \\
\hline Registra o contato do usuário para avisar quando o medicamento chegar & 01 & 4 \\
\hline Orienta ao usuário a retornar ao prescritor & 13 & 52 \\
\hline Não respondeu & 01 & 4 \\
\hline
\end{tabular}

Fonte: Autores.

Conforme demonstrado na Tabela 2, foi relatado por $40 \%$ dos profissionais que, sempre há falta medicamentos em suas Unidades e que $80 \%$ adotam a conduta de relatar ao paciente que o medicamento está em falta. Outra conduta muito recorrente mostra que $76 \%$ orientam ao usuário a procurarem o programa Nacional “Aqui tem farmácia popular” e apenas $4 \%$ registra o contato do paciente para avisá-lo quando o medicamento estará disponível para dispensação.

Durante a entrevista, alguns motivos para a falta de medicamentos nas Unidades foram relatados pelos profissionais, dentre eles, destacam-se a falta de recursos humanos " $n$ ão dispõe de auxiliares de farmácia suficientes para o gerenciamento de estoque no almoxarifado e no abastecimento na parte física da farmácia", falhas na programação de medicamentos “medicamentos que foram solicitados mas não foram atendidos”, " a aquisição dos medicamentos prestes a vencer o prazo de validade", problemas relacionados ao Sistema Hórus "falta de internet para alimentação de dados". Desse modo, a falta de auxiliares implica na atribulação ao farmacêutico, bem como, no controle de estoque e na deficiência no abastecimento de medicamentos na parte física da farmácia, afetando o acesso destes, pelos usuários.

Segundo Melo e Castro (2017), uma das soluções para reduzir a falta de medicamentos, seria a gestão adequada do estoque de medicamentos, resultando em redução do desperdício e permitir uma programação adequada para a distribuição do medicamento do almoxarifado à Unidade, bem como, a inclusão de novos medicamentos na Relação Municipal de Medicamentos Essenciais (REMUME).

No que diz respeito às atividades de dispensação de medicamentos nas UBS, quanto a orientação ao usuário na hora da dispensação, evidenciou-se que 32\% dos profissionais farmacêuticos responderam que sempre orientam o usuário a forma de como utilizá-los e $40 \%$ relataram que às vezes, somente quando é solicitado. Já em relação às queixas técnicas ou notificações de eventos adversos (EA) por medicamentos, $84 \%$ dos profissionais relataram que não ocorreu tal situação na Unidade e não 
fizeram nenhum tipo de procedimento, entretanto, $8 \%$ assumiram que houve reclamação e que utilizaram o sistema VigiMed, ferramenta disponibilizada pela ANVISA (2020), bem como, esse registro também é notificado no Procedimento Operacional Padrão (POP) adotado pela própria Unidade (Tabela 03).

Tabela 3 - Atividades realizadas na dispensação de medicamentos nas UBS (N=25), Belém-Pará, 2020.

\begin{tabular}{lcc}
\hline $\begin{array}{l}\text { Orientação ao usuário sobre o uso do medicamento na hora da } \\
\text { dispensação }\end{array}$ & N & \% \\
\hline Sempre & 8 & 32 \\
Repetidamente & 5 & 20 \\
Às vezes & 10 & 40 \\
Raramente & 2 & 8 \\
Nunca & & 8 \\
\hline Registro de notificação de queixas técnicas e/ou eventos adversos & & 84 \\
Sim & 2 & 8 \\
Não & 21 & 2 \\
Não responderam & 2 & \\
\hline
\end{tabular}

Fonte: Autores.

É válido ressaltar que, durante a queixa técnica de EA, foi relatado pelo profissional farmacêutico que, "foi realizada a orientação aos usuários, a suspenderem os medicamentos e voltarem ao médico para reverem a terapia medicamentosa e investigar o problema citado".

Alguns estudos apontam que as intervenções farmacêuticas, entre elas a orientação no momento da dispensação tem se mostrado positiva na melhora da hipertensão arterial, reduzindo custos, melhorando as prescrições, controlando a possibilidade de eventos adversos e promovendo maior adesão do paciente ao tratamento (Melo \& Castro, 2017). Segundo Ribeiro e colaboradores (2015), em seu estudo mostraram que a avaliação da prescrição médica pelo farmacêutico resultou no aumento de oportunidades de intervenções por parte deste profissional, resultando na eficácia do tratamento farmacológico. A Organização Mundial de Saúde (OMS) preconiza que o farmacêutico destine à orientação ao menos três minutos por paciente. No estudo de Oenning e colaboradores (2011), realizado no município de Grão Pará, no estado de Santa Catarina, a duração da dispensação foi de 2,6 minutos por paciente, superior à média de outros estudos, como na pesquisa de Naves e Silver (2005), realizada em 15 centros de saúde, no Distrito Federal, que teve tempo médio dispensação que durou 52,3 segundos; e de um outro estudo realizado em Ribeirão Preto, em dez UBS, esse tempo foi estimado em 18,4 segundos (Santos \& Nitrini, 2004). Deste modo, observa-se que a dispensação requer uma atenção maior por parte do profissional farmacêutico, visto que, o mesmo por desenvolver outras atividades administrativas gerenciais, não possui disponibilidade adequada para se dedicar a escuta e orientação ao usuário, obtendo impactos diretos na resolutividade do tratamento prescrito.

Em relação às atividades de ações clínico-assistenciais, notou-se que somente três UBS prestam serviços clínicos farmacêuticos. Dentre as abordagens executadas pelos farmacêuticos ao praticarem as atividades de atuação clínica, evidenciouse que são realizadas em maiores percentuais $(100 \%)$, a prática da consulta farmacêutica e o acompanhamento 
farmacoterapêutico e em menor percentual $(66,67 \%)$ o serviço de reconciliação de medicamentos, conforme descrita na Tabela 4.

Tabela 4 - Abordagem executada pelos profissionais farmacêuticos nas UBS (N=03) ao desenvolverem serviços clínicos farmacêuticos, Belém-Pará, 2020.

\begin{tabular}{llc}
\hline Abordagem & N & $\%$ \\
\hline Consulta farmacêutica & 3 & 100,0 \\
Acompanhamento farmacoterapêutico & 3 & 100,0 \\
Reconciliação de medicamentos & 2 & 66,67 \\
\hline
\end{tabular}

Fonte: Autores.

Podemos encontrar na literatura experiências sobre implantação do cuidado farmacêutico na APS, demonstrando ofertas simultâneas de serviços clínicos farmacêuticos com resultados significativos, evidenciando um aumento na adesão ao tratamento e consequentemente uma melhora nas condições de saúde dos usuários (Cff, 2016a; Brasil, 2019). Nas UBS em estudo, segundo os profissionais farmacêuticos, ao realizarem a prática da consulta farmacêutica e o acompanhamento farmacoterapêutico, observaram resultados positivos significativos como: redução de problemas relacionados a medicamentos (PRMs), diminuição da polifarmácia, diminuição de EA e uma melhor adesão terapêutica pelos usuários. Logo, a presença desse profissional no cuidado centrado ao paciente na APS, contribui para o uso racional de medicamentos e para uma maior efetividade no tratamento medicamentoso, impactando dessa forma, em uma maior resolutividade das ações em saúde.

No que tange a oferta dessas atividades clínicas abordadas por esses profissionais, observou-se que em maior proporção são oferecidos a um grupo específico de usuários $(66,67 \%)$ vinculados ao Programa HIPERDIA (acompanhamento de portadores de hipertensão arterial e/ou diabete mellitus). A oferta do serviço a todos os usuários de saúde que procuram ou necessitam, está disponível em apenas 33,33\% das UBS (Tabela 5). 
Tabela 5 - Atividades clínicas x Critério de priorização pelas UBS (N=03), Belém-Pará, 2020.

\begin{tabular}{llc}
\hline Atividades Clínicas & N & $\%$ \\
\hline Oferta dos Serviços Clínicos farmacêuticos & & 33,33 \\
A todos os usuários da unidade de Saúde & 1 & 33,33 \\
Apenas quando é solicitado pelo usuário & 1 & 66,67 \\
A um grupo específico & 2 & 33,33 \\
Quando o usuário apresenta algum tipo de dificuldade com o uso & 1 & 66,67 \\
do medicamento & & 33,33 \\
\hline Critérios para priorização dos pacientes quanto as \\
necessidades dos serviços de farmácia clínica
\end{tabular}

Fonte: Autores.

A disponibilização do serviço de forma mais centralizada a grupos com morbidade de doenças crônicas, pode estar diretamente correlacionada ao fato de serem usuários que fazem uso contínuo de medicamentos, estando mais predispostos a um número maior de EA e ao uso de programas assistenciais para a aquisição de medicamentos e controle dos sintomas, além da queixa principal de outros profissionais que atuam na equipe de saúde sobre a falta de adesão medicamentosa aos mesmos. Vale ressaltar que o atendimento disponível para todos os usuários, realizado em uma Unidade, "se dá principalmente em função da baixa escolaridade da comunidade" onde fica localizada esta UBS, relato feito pela própria farmacêutica. Para minimizar estes transtornos é realizado educação em saúde através de parcerias pré-estabelecidas com instituições de ensino superior, através de estágios voluntários e bolsistas do Programa de Educação pelo Trabalho para Saúde (PET-Saúde), visando contribuir para o conhecimento dos usuários sobre determinada doença e como preveni-la.

No estudo, de Santos, Monteiro e Damascena (2020), foi demonstrada a importância da interação da equipe multidisciplinar na AB, no cuidado efetivo a grupos de doenças crônicas, por meio de acompanhamento e atividade de educação em saúde, corroborando para o aumento de adesão desses pacientes ao tratamento, contribuindo dessa forma, para ações de promoção à saúde e prevenção de doenças.

Pereira e colaboradores (2017), afirmam que alguns estados que já realizaram a implantação dos serviços clínicos farmacêuticos, com ênfase em pacientes hipertensos e diabéticos, geraram bons resultados. Entretanto, tal prática, ainda não está presente em todo Brasil. Fato este, que pode estar correlacionado ao excesso de atividades administrativas sob a responsabilidade do farmacêutico, em detrimento ao cuidado efetivo dos usuários (Araújo, P. et al.; Araújo, S. et al., 2017).

No que diz respeito aos registros relacionados aos Sefar, notou-se que 66,67\% dos profissionais possuem registro próprio (Prontuário do paciente) e são arquivados na própria farmácia, assim como, registram no sistema informatizado através de planilhas. Já o registro no prontuário da Unidade, apresentou baixo percentual, 33,33\%. Não se obteve respostas as opções assinaladas com X, conforme descrito no Quadro 1. 
Quadro 1 - Registro dos serviços clínicos farmacêuticos nas UBS (N=03), Belém-Pará, 2020.

\begin{tabular}{|l|c|c|c|}
\hline & Sim & Não & N(\%) \\
\cline { 2 - 4 } & Prontuário da unidade & $\mathrm{X}$ & $\mathbf{N}(\%)$ \\
\hline $\begin{array}{l}\text { Registro próprio arquivado } \\
\text { na farmácia }\end{array}$ & $2(33,33)$ & $\mathrm{X}$ & $\mathrm{X}$ \\
\hline Sistema informatizado & $2(66,67)$ & $\mathrm{X}$ & $\mathrm{X}$ \\
\hline Outro & $2(66,67)$ & $\mathrm{X}$ & $\mathrm{X}$ \\
\hline
\end{tabular}

Fonte: Autores.

É válido ressaltar, que o registro próprio, arquivado na farmácia, pode dificultar a circulação das informações entre a equipe multiprofissional envolvida com o cuidado ao usuário (Araújo, S. et al., 2017). Deste modo, é preferível que o profissional farmacêutico registre os serviços clínicos no prontuário da própria unidade, pois as trocas de informações entre a equipe de saúde podem contribuir para o uso seguro e racional de medicamentos e para uma melhor continuidade e efetividade do cuidado.

Dentre os principais motivos que justificam a ausência dos serviços clínicos farmacêuticos, nas demais Unidades $(\mathrm{N}=22)$, identificou-se problemas relacionados à estrutura, do qual 86,36\% responderam que não dispõe de espaço físico para a realização desse serviço, já 68,18\% relataram deficiência de recursos humanos, enquanto 50\% dos profissionais, justificaram a indisponibilidade de tempo. A falta de apoio do gestor municipal e o espaço físico inadequado obteve apenas 31,82\% (Gráfico 2).

Gráfico 2 - Motivos que justificam a ausência dos Serviços clínicos nas UBS (N=22), Belém-Pará, 2020.

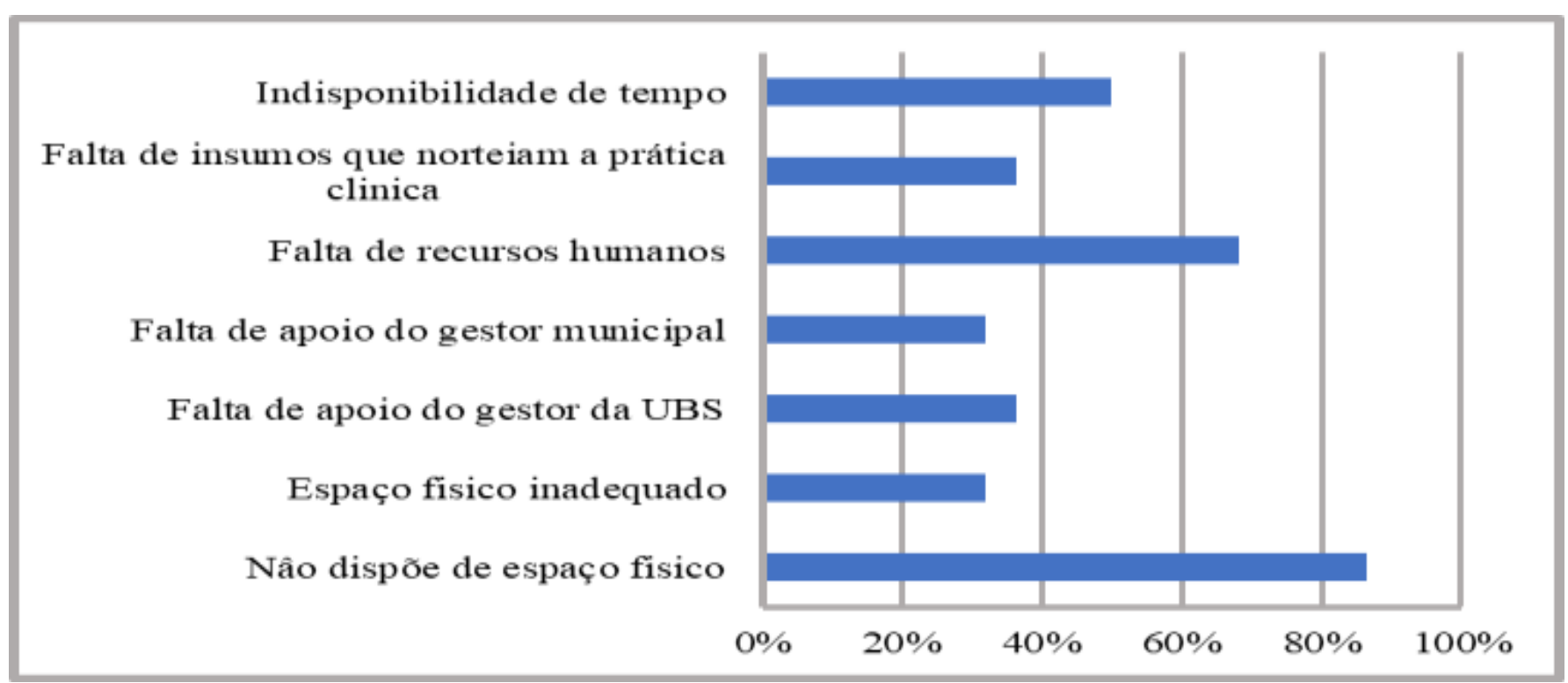

Fonte: Autores.

No estudo realizado por Araújo e colaboradores (2017), os principais motivos pelos quais os farmacêuticos alegam não conseguir realizar atividades clínicas, seriam por indisponibilidade de tempo, espaço físico, falta de incentivo do município, e/ou 
por pessoal insuficiente na farmácia. A ausência de tais estruturas inviabiliza ou restringe o desenvolvimento dos serviços clínicos farmacêuticos de forma mais abrangente, visto que, não ter condições de atender um usuário com individualidade e conforto ou com barreiras físicas dificulta a comunicação efetiva e a relação terapêutica. $\mathrm{O}$ atendimento através de janelas ou grades, separando os usuários dos responsáveis pela dispensação, é um problema organizacional que pode ter importantes reflexos na terapêutica, além de prejudicar a comunicação com pacientes idosos, gestantes e usuários com necessidades especiais, já que a humanização do atendimento ao usuário tem sido associada à adesão ao tratamento prescrito (Leite, et al.; Barbosa, et al., 2017).

Em relação sobre as legislações que dão amparo legal às atribuições clínicas do profissional farmacêutico, foram citadas com maior frequência as legislações RDC no 585 e a RDC n 586 de 2013, com 56\% e 48\% respectivamente. Já 36\% dos profissionais farmacêuticos não souberam responder, e a RDC no 499 de 2009 não foi mencionada por nenhum farmacêutico, conforme descrita na Tabela 6.

Tabela 6: Legislações que dão amparo legal as atividades de caráter clínico, Belém-Pará, 2020.

\begin{tabular}{|c|c|c|}
\hline Legislação & $\mathbf{N}$ & $\%$ \\
\hline \multicolumn{3}{|l|}{$\mathrm{RDC} \mathrm{n}^{\circ} 499$ de 2008} \\
\hline RDC nº 44 de 2009 & 1 & 4 \\
\hline $\mathrm{RDC} \mathrm{n}^{\circ} 585$ de 2013 & 14 & 56 \\
\hline $\mathrm{RDC} \mathrm{n}^{\circ} 586$ de 2013 & 12 & 48 \\
\hline Lei $\mathrm{n}^{\circ} 13.021$ de 2014 & 7 & 28 \\
\hline Não sei & 3 & 12 \\
\hline Não responderam & 6 & 24 \\
\hline
\end{tabular}

Fonte: Autores.

A farmácia clínica não deve ser considerada apenas como uma reação à industrialização, e sim como uma resposta à necessidade social no sentido de garantir a segurança do uso de medicamentos. Estudos presentes em revisões sistemáticas, mostram que o farmacêutico influencia positivamente nos resultados da farmacoterapia através da Atenção Farmacêutica, assumindo o papel do cuidado ao paciente, o qual requer um conhecimento adequado, habilidades de comunicação, capacidade para resolver problemas, tomada de decisões, e habilidades de raciocínio crítico (Freitas, et al., 2016; Araújo, S. et al., 2017). Neste contexto, fica evidente, que é essencial que o profissional farmacêutico tenha amplo conhecimento dessas legislações, pois são indispensáveis para a prestação dos serviços clínicos farmacêuticos aos usuários, a fim de garantir a estes, uma assistência integral, segura e de qualidade.

\section{Considerações Finais}

A proposta executada é uma estratégia inicial para subsidiar posterior implantação do serviço da consulta farmacêutica na rede municipal de saúde de Belém, haja vista as evidências por meio do diagnóstico situacional que comprovam a ausência do serviço de modo padronizado em detrimento a necessidade pelos usuários da $\mathrm{AB}$ em Belém/PA. É válido ressaltar, que uma das dificuldades encontradas para a implantação da consulta farmacêutica nas UBS no município é a indisponibilidade de tempo do profissional. No entanto, uma das estratégias para iniciar a implantação da prática clínica nas UBS estudadas, seria a 
implementação de um serviço que demandasse menos tempo para o profissional farmacêutico e menor complexidade, dentre elas podemos citar: a dispensação e a orientação. Dessa forma, o profissional farmacêutico pode contribuir incisivamente para o cuidado efetivo centrado no paciente, proporcionando a este uma melhor qualidade de vida. Dada a importância ao tema, tornase necessário o desenvolvimento de projetos que visem a educação continuada dos farmacêuticos, voltados para as práticas clínico-assistenciais e a para ampliação de projetos em parceria com as Universidades, com a finalidade de desencadear competências e habilidades à comunidade acadêmica de farmácia, bem como contribuir para sua formação profissional. O referido estudo, visa contribuir com o fortalecimento das políticas de saúde e com o cuidado integral aos usuários da APS da região metropolitana de Belém.

\section{Agradecimentos}

Agradecimentos à Faculdade de Farmácia da Universidade Federal do Pará (UFPA), a Secretária Municipal de Saúde de Belém e aos seus colaboradores da Farmácia da Rede Básica de Saúde, pelo apoio e de disponibilizar seu tempo para agregar na pesquisa.

\section{Referências}

Agência Nacional de Vigilância Sanitária. https://www.gov.br/anvisa/pt-br/assuntos/noticias-anvisa/2019/vigimed.

Akerman, M., \& Freitas, O. D. (2017). Pesquisa Nacional sobre Acesso, Utilização e Promoção do Uso Racional de Medicamentos (PNAUM): avaliação dos serviços de atenção farmacêutica primária.

Andreoli, G. L. M., \& Dias, C. N. (2015). Planejamento e gestão logística de medicamentos em uma central de abastecimento farmacêutico hospitalar. RAHISRevista de Administração Hospitalar e Inovação em Saúde, 12(4).

Araújo, P. S., Costa, E. A., Guerra Junior, A. A., Acurcio, F. D. A., Guibu, I. A., Álvares, J., \& Leite, S. N. (2017). Atividades farmacêuticas de natureza clínica na atenção básica no Brasil. Revista de Saúde Pública, 51, 6s.

Araújo, S. Q., Costa, K. S., Luiza, V. L., Lavras, C., Santana, E. A., \& Tavares, N. U. L. (2017). Organização dos serviços farmacêuticos no Sistema Único de Saúde em regiões de saúde. Ciência \& Saúde Coletiva, 22, 1181-1191.

Arruda, C., Lopes, S. G. R., Koerich, M. H. A. D. L., Winck, D. R., Meirelles, B. H. S., \& Mello, A. L. S. F. D. (2015). Redes de atenção à saúde sob a luz da teoria da complexidade. Escola Anna Nery, 19(1), 169-173.

Barbosa, M. M., Garcia, M. M., Nascimento, R. C. R. M. D., Reis, E. A., Guerra Junior, A. A., Acurcio, F. D. A., \& Álvares, J. (2017). Avaliação da infraestrutura da assistência farmacêutica no sistema único de saúde em Minas Gerais. Ciência \& Saúde Coletiva, 22, $2475-2486$.

Barbosa, P. (2018). Farmacêuticos da Sesma passam por qualificação visando à ampliação do atendimento. http://agenciabelem.com.br/Noticia/165332/farmaceuticos-da-sesma-passam-por-qualificacao-visando-a-ampliacao-do-atendimento

Barros, D. S. L., Silva, D. L. M., \& Leite, S. N. (2020). Serviços farmacêuticos clínicos na atenção primária à saúde do brasil. Trabalho, Educação e Saúde, 18(1).

Costa, K. S., \& Nascimento Jr, J. M. (2012). HÓRUS: inovação tecnológica na assistência farmacêutica no Sistema Único de Saúde. Revista de Saúde Pública, 46, 91-99. https://www.scielo.br/pdf/rsp/2012nahead/ao4223.pdf

Costa, K. S., Tavares, N. U. L., Nascimento Júnior, J. M. D., Mengue, S. S., Álvares, J., Guerra Junior, A. A., \& Soeiro, O. M. (2017). Assistência farmacêutica na atenção primária: a pactuação interfederativa no desenvolvimento das políticas farmacêuticas no Sistema Único de Saúde (SUS). Revista de Saúde Pública, 51, 2s.

Da Cruz, W. M., de Queiroz, L. M. D., \& Soler, O. (2020). Cuidado farmacêutico para utentes de farmácia comunitária privada: Revisão sistemática. Brazilian Journal of Development, 6(10), 78682-78702.

Dal Molin, S. S., Borges, M. S., \& Picolo, J. D. (2018). Análise dos custos e formação do preço de venda da prestação de serviços farmacêuticos em uma farmácia comunitária. In Congresso Sul Catarinense de Administração e Comércio Exterior (Vol. 2).

De Farmácia, C. F. (2016a). Serviços farmacêuticos diretamente destinados ao paciente, à família e à comunidade: contextualização e arcabouço conceitual: Conseho Federal de Farmácia, 200.

De Farmácia, C. F. (2016b). Programa de Suporte ao Cuidado Farmacêutico na Atenção à Saúde (PROFAR); Conselho Federal de Farmácia.

De Freitas, G. R. M., Luna-Leite, M. D. A., De Castro, M. S., \& Heineck, I. (2016). Principais dificuldades enfrentadas por farmacêuticos para exercerem suas atribuições clínicas no Brasil. Revista Brasileira de Farmácia Hospitalar e Serviços de Saúde, 7(3). 
De Lima, É. D., Da Silva, R. G., Ricieri, M. C., \& Blatt, C. R. (2017). Farmácia clínica em ambiente hospitalar: enfoque no registro das atividades. Revista Brasileira de Farmácia Hospitalar e Serviços de Saúde, 8(4).

Lansing, A., de Souza, J., Fernandes, L. C., de Castro, L. C., \& Kauffmann, C. (2017). O farmacêutico em serviço de atenção secundária à saúde: atuação em equipe multiprofissional para promoção do uso racional de medicamentos. Revista Destaques Acadêmicos, 9(3).

Leite, S. N., Manzini, F., Álvares, J., Guerra Junior, A. A., Costa, E. A., Acurcio, F. D. A., \& Farias, M. R. (2017). Infraestrutura das farmácias da atenção básica no Sistema Único de Saúde: análise dos dados da PNAUM-Serviços. Revista de Saúde Pública, 51, 13s.

Luquetti, T. M., Santos, J. B., Bitencourt, G. R., Castilho, S. R., \& Elias, S. C. (2017). Serviços farmacêuticos na atenção primária à saúde: Percepção dos farmacêuticos. Diversitates Int $J, 09$ (3).

Mattos, L.V. (2015). Assistência farmacêutica na atenção básica e Programa Farmácia Popular do Brasil: uma análise crítica das políticas públicas de provisão de medicamentos no Brasil. Dissertação (Saúde Pública). https://www.arca.fiocruz.br/handle/icict/14059

Melo, D. O. D., \& Castro, L. L. C. D. (2017). Pharmacist's contribution to the promotion of access and rational use of essential medicines in SUS. Ciencia \& saude coletiva, 22, 235-244.

Ministério da Saúde (2015). Cuidado farmacêutico na atenção básica: serviços farmacêuticos na atenção básica à saúde. Resultados do projeto de implantação do cuidado farmacêutico no Município de Curitiba. http://bvsms.saude.gov.br/bvs/publicacoes/resultado_projeto_implantacao_cuidados_farmaceuticos.pdf

Ministério da Saúde (2016a). Programa Nacional de Qualificação da Assistência Farmacêutica no Âmbito do Sistema Único de Saúde (QUALIFARSUS). Eixo estrutura atenção básica: Instruções Técnicas. http://bvsms.saude.gov.br/bvs/publicacoes/qualifarsus_programa_nacional_qualificacao_farmaceutica.pdf

Ministério da Saúde (2016b). Componente Avaliação dos Serviços de Assistência Farmacêutica Básica: introdução, método e instrumentos. Serie PnaumPesquisa Nacional sobre o Acesso, Utilização e promoção do Uso Racional de Medicamentos no Brasil. http://bvsms.saude.gov.br/bvs/publicacoes/componente_populacional_introducao_metodo_instrumentos.pdf

Ministério da Saúde (2017). Portaria n 2.436, de 21 de setembro de 2017. Aprova a Política Nacional de Atenção Básica, estab elecendo a revisão de diretrizes para a organização da Atenção Básica, no âmbito do Sistema Único de Saúde (SUS). https://bvsms.saude.gov.br/bvs/saudelegis/gm/2017/prt2436_22_09_2017.html

Ministério da Saúde (2019). Secretaria de atenção primária à saúde. Departamento de saúde da família. Gestão do Cuidado Farmacêutico na Atenção Básica. http://189.28.128.100/dab/docs/portaldab/publicacoes/Livro_Atencao_basica_Farmaceutica_SET2019.pdf

Naves, J. D. O. S., \& Silver, L. D. (2005). Avaliação da assistência farmacêutica na atenção primária no Distrito Federal. Revista de Saúde Pública, 39(2), 223230 .

Nicoletti, M. A., \& Ito, R. K. (2018). Formação do farmacêutico: novo cenário de atuação profissional com o empoderamento de atribuições clínicas. Revista Saúde-UNG-Ser, 11(3/4), 49-62.

Oenning, D., Oliveira, B. V. D., \& Blatt, C. R. (2011). Conhecimento dos pacientes sobre os medicamentos prescritos após consulta médica e dispensação. Ciência \& Saúde Coletiva, 16, 3277-3283.

Pereira, A. S., Shitsuka, D. M., Parreira, F. J., \& Shitsuka, R. (2018). Metodologia da pesquisa científica. [e-book]. Santa Maria. Ed. UAB/NTE/UFSM. https://repositorio. ufsm. br/bitstream/handle/1/15824/Lic_Computacao_Metodologia-Pesquisa-Cientifica. pdf.

Pereira, M. G., Prado, N. M. D. B. L., \& Krepsky, P. B. (2017). Resultados de seguimento farmacoterapêutico a pacientes hipertensos em farmácia comunitária privada na Bahia. Revista Baiana de Saúde Pública, 41(2).

Pereira, N. C., Luiza, V. L., \& Cruz, M. M. D. (2015). Serviços farmacêuticos na atenção primária no município do Rio de Janeiro: um estudo de avaliabilidade. Saúde em Debate, 39, 451-468.

Prefeitura Municipal de Belém. http://www.belem.pa.gov.br.

Ribeiro, V. F., Sapucaia, K. C. G., Aragão, L. A. O., Bispo, I. C. D. S., Oliveira, V. F., \& Alves, B. L. (2015). Realização de intervenções farmacêuticas por meio de uma experiência em farmácia clínica. Revista Brasileira de Farmácia Hospitalar e Serviços de Saúde, 6(4).

Rossignoli, P., Pontarolli, D. R. S., Corrêa, L. G. P., de Lima Germano, J., Pontarolo, R., \& Fernandez-Llimos, F. (2019). Inovação em serviços farmacêuticos clínicos no componente especializado da assistência farmacêutica do Estado do Paraná. Revista de Saúde Pública do Paraná, 2(1), 125-139.

Santos, D. de M., Monteiro, C. M. de O., \& Damascena, C. G. The characterization of health groups implemented by family health teams and Nasf-AB in a regional in the Municipality of Jaboatão dos Guararapes. Research, Society and Development, 09 (12). 10.33448/rsd-v9i12.11160. https://rsdjournal.org/index.php/rsd/article/view/11160.

Santos, V. D., \& Nitrini, S. M. (2004). Indicadores do uso de medicamentos prescritos e de assistência ao paciente de serviços de saúde. Revista de Saúde Pública, 38(6), 819-834.

Secretaria de ciências, Tecnologias e Insumos Estratégicos (2014). Departamento de Assistência Farmacêutica e Insumos Estratégicos. Cuidado Farmacêutico na Atenção básica. Capacitação para implantação dos serviços de clínica farmacêutica. http://bvsms.saude.gov.br/bvs/publicacoes/cuidado_farmaceutico_atencao_basica_saude_2.pdf

Silva, D. Á. M., Mendonça, S. D. A. M., Oliveira, D. R. D., \& Chemello, C. (2018). A prática clínica do farmacêutico no núcleo de apoio à saúde a família. Trabalho, Educação e Saúde, 16(2), 659-682. 
Research, Society and Development, v. 10, n. 1, e33310111803, 2021

(CC BY 4.0) | ISSN 2525-3409 | DOI: http://dx.doi.org/10.33448/rsd-v10i1.11803

Soler, O., Sinimbu, A. V., de Figueredo, D. C., dos Santos Vieira, H. K., da Rocha Galucio, N. C., Pinheiro, P. D. N. Q., \& Ledo, Y. (2017). A Reorientação Da Assistência Farmacêutica Na Secretaria Municipal De Saúde De Belém (Pa): Relato de experiência. Revista Eletrônica de Farmácia, 14(4). 